\title{
Prediction of degree of crystallinity for the LTA zeolite using artificial neural networks
}

\author{
SHAHRAM GHANBARI ${ }^{1}$, BEHZAD VAFERI ${ }^{2, *}$ \\ ${ }^{1}$ Department of Chemical Engineering, Malek-Ashtar University of Technology, Tehran, Iran \\ ${ }^{2}$ Department of Chemical Engineering, Shiraz Branch, Islamic Azad University, Shiraz, Iran
}

\begin{abstract}
Zeolites are microporous aluminosilicate/silicate crystalline materials with three-dimensional tetrahedral configuration. In this study, the degree of crystallinity of the synthesized Linde Type A (LTA) zeolite, which is the main indicator of its quality/purity is tried to be modeled. Effect of crystallization time, temperature, molar ratio of the synthesis gel on the relative crystallinity of the LTA zeolites is investigated using artificial neural networks. Our experimental observations and some data collected from literature have been used for adjusting the parameters of the proposed model and evaluating its performance. It has been observed that two-layer perceptron network with eight hidden neurons is the most accurate approach for the considered task. The designed model predicts the experimental datasets with a mean square error of $3.99 \times 10^{-6}$, absolute average relative deviation of $8.69 \%$, and regression coefficient of 0.9596 . The proposed model can decrease the required time and number of experiments to evaluate the extent of crystallinity of the LTA zeolites.
\end{abstract}

Keywords: LTA zeolite; relative crystallinity; artificial intelligence approach; optimum topology

\section{Introduction}

Zeolites play an important role as catalyst [13], ion exchanger [4], membrane [5], gas separation [6], and photo-catalytic degradation of materials [7]. These crystalline materials may be produced naturally or synthesized in laboratory [811]. In order to synthesize a specific type of zeolite, appropriate ingredients should be used, their ratio in the reaction mixture should be accurately adjusted, and special attention should be given to the temperature and crystallization time [12].

Chemically controlled particulate properties of zeolites [13], kinetics of zeolite crystallization [14], effect of thermal and hydrothermal treatment [15], influence of anions on the kinetics of zeolite crystallization [16], growth mechanism [17], and heating methods [18, 19] are the most popular aspects of zeolites which have extensively been investigated by different researchers. In spite of a large amount of experimental studies on zeolites, they have not been effectively investigated from

*E-mail: behzad.vaferi@gmail.com the theoretical point of view, and mathematical simulations of their behavior have not been fully developed.

Large number of experimental data on synthesis of the Linde Type A (LTA) zeolite [10, 12, 20-23], and importance of simulation of its theoretical behavior motivated us to study this route from different points of view. Therefore, a focus of the present research is concentrated on designing an intelligent tool to study the effect of the most influential synthesis parameters on the relative crystallinity of the LTA zeolites.

Experimental analyses confirm that the degree of relative crystallinity of the LTA zeolites depends mainly on the concentration of components in reaction mixture, operating parameters, and synthesis method [13, 24-26]. Initial silicon dioxide $\left(\mathrm{SiO}_{2}\right)$, aluminum oxide $\left(\mathrm{Al}_{2} \mathrm{O}_{3}\right)$, sodium oxide $\left(\mathrm{Na}_{2} \mathrm{O}\right)$ and water content in the reaction mixture, crystallization time, aging time, stirring speed, temperature, and heating method are the parameters which have a substantial influence on the degree of relative crystallinity of the LTA zeolites [13, 24-26]. 
Although it is possible to take into account all of the above mentioned parameters, attention of the present study has been concentrated on a development of a straightforward, simple and practical model for the considered task. Therefore, only those key variables that have strong influence on the degree of crystallinity of the LTA zeolite have been chosen as the independent variables. The molar ratio of the ingredients in the synthesis mixture $\left(\mathrm{SiO}_{2}: \mathrm{Al}_{2} \mathrm{O}_{3}, \mathrm{Na}_{2} \mathrm{O}: \mathrm{SiO}_{2}, \mathrm{H}_{2} \mathrm{O}: \mathrm{Na}_{2} \mathrm{O}\right)$, crystallization time, and temperature were used as independent variables for estimating the extent of crystallinity of the LTA zeolites. It should be mentioned that in order to decrease the number of independent variables, instead of using concentration of synthesized ingredients, their molar ratios were employed. Our experimental observations as well as experimental data reported by six different groups of researchers $[10,12,20-23]$ have been utilized for designing the Artificial Neural Networks (ANN) model for simulating the degree of crystallinity of LTA zeolites.

In the subsequent sections, a brief description of the ANN approach, its different types, and procedure of finding its optimal topology are presented. Various ANN types have been designed, their predictive accuracies have been compared using some statistical indices, and the best one has been selected.

\section{Mathematical model}

\subsection{Artificial neural networks}

Artificial neural networks (ANN) are developed by mathematical simulation of behavior of the human neural system [27-30]. Excellent performance of the ANN approaches in tracking behavior of various scientific problems, combined with their simplicity and flexibility have led to high popularity of these intelligent and non-linear methods in the recent years [31-33]. These methodologies often have at least two layers composing a number of processing nodes, namely neurons. The neurons of each layer may interconnect to the neurons of the previous or subsequent layer(s) with respect to the type of ANN model. Various types of mathematical models can be employed as a practical tool for pattern recognition [33], function estimation [34, 35], fault detection [36] and also be a powerful technique for optimization of various processes [37].

It should be mentioned that training of these smart artificial networks must be done by an appropriate learning method. By training the ANN model, it is possible to relate dependent and independent variables of the most nonlinear multivariable systems with arbitrary complexity [2730]. Back propagation, cascade correlation, and conjugate gradient are the most popular learning algorithms [38].

\subsection{Different types of artificial neural net- works}

\subsubsection{Feed-forward ANN models}

The ANN models may be classified by the way that the neurons of each layer are connected to the neurons of previous or/and subsequent layers. Feed-forward and recurrent networks are two different types of the ANN models [39, 40]. The feedforward topologies including multi-layer perceptron (MLP), radial basis function (RBF), generalized regression (GR), and cascade-forward backpropagation (CFB) neural networks are among the most widely used types of ANN models for handling the regression problem [30, 33, 41, 42]. In spite of neurons of the CFB network that are connected to the neurons of all of the subsequent layers, neurons of the MLP, RBF and GR networks are only connected to the neurons of the subsequent layer [30, 33, 41, 42]. Since the MLP network often shows better performance in modeling and predicting behavior of various phenomena, it has been studied in more detail in this section.

As Fig. 1 illustrates, the MLP network is composed of input, hidden and one output layer. Independent variables enter the hidden layer. This layer performs some mathematical manipulations on the received information and then transfers it to the output layer [40]. The neuron(s) of the output layer present values of the dependent variables. The output value from each artificial neuron can be computed using equation 1 : 


$$
n_{j}=f\left(\sum_{r=1}^{N} w_{j r} x_{r}+b_{j}\right)
$$

Equation 1 shows that the output of a given neuron can be calculated by: (1) algebraic addition of bias coefficients $b_{j}$ to the multiplication of entered information $\mathrm{x}_{\mathrm{r}}$ and its particular weight coefficients $\mathrm{w}_{\mathrm{jr}}$, and (2) passing the value of stage one through a special function, namely transfer function $\mathrm{f}$. Equation 2 and equation 3 which represent the hyperbolic tangent and logarithmic sigmoid respectively, are the most commonly used transfer functions.

$$
\begin{gathered}
f(x)=\frac{\exp (x)-\exp (-x)}{\exp (x)+\exp (-x)} \\
f(x)=\frac{1}{1+\exp (-x)}
\end{gathered}
$$

Continuity, nonlinearity and differentiability of these transfer functions are the main characteristics that allow the ANN model to relate independent and dependent variables of a considered phenomenon [37, 38].

\subsubsection{Recurrent ANN models}

Elman [39] and Hopfield [43] are the most wellknown types of the recurrent networks. The Elman network is a two-layer MLP network in which the neurons of the first layer have both forward and backward connections [39]. All of the neurons of the Hopfield network are completely interconnected, and therefore this ANN type has the maximum number of connections among the networks with equal number of neurons [43].

\subsection{Optimum structure of ANN model}

Determining the optimum number of hidden layers, and number of neurons, in each hidden layer there are two important steps for designing the ANN-based approaches.

\subsubsection{Optimum number of hidden layers}

It has been scientifically confirmed that the MLP network with a single hidden layer having a non-linear transfer function can accurately simulate the behavior of majority of multivariable processes [44-46]. Therefore, in the present research the MLP network with only one hidden layer is also employed for estimating the amount of crystallinity of the LTA zeolites.

\subsubsection{Optimum number of hidden neurons}

An ideal number of hidden neurons is not known at all, and often determined by trial and error process [29, 30]. Large numbers of hidden neuron suffer from an over-fitting, and often need high computation efforts, while small numbers of hidden neuron are unable to relate dependent to independent variables with an acceptable accuracy [33-35].

Network growing and network pruning are two effective strategies for evaluation an optimum number of hidden neurons [47]. Network pruning commences with a large number of hidden units, and tries to remove the extra neurons during the training stage, while the network growing strategy starts with a small network and increases the number of hidden neurons until a desired accuracy is achieved [48]. Since the majority of learning effort of pruning algorithms are allocated to the networks which are bigger than necessary, the network growing strategy often presents more efficient performance.

In summary, it can be said that a MLP network with random structure (number of hidden layers as well as hidden neurons) often encounters severe difficulties in modeling and simulating the behavior of considered processes.

\section{Results and discussion}

\subsection{Design of an ANN model}

Based on the previous explanations, only some easily measured variables, i.e. molar ratio of the $\mathrm{SiO}_{2}: \mathrm{Al}_{2} \mathrm{O}_{3}, \mathrm{Na}_{2} \mathrm{O}: \mathrm{SiO}_{2}$ and $\mathrm{H}_{2} \mathrm{O}: \mathrm{Na}_{2} \mathrm{O}$ in the reaction mixture, temperature and crystallization time are considered as independent variables for estimation an extent of relative crystallinity of the LTA zeolites by the different ANN approaches.

The schematic of the developed MLP model for prediction the relative crystallinity of the LTA zeolites is shown in Fig. 1. As Fig. 1 shows, our developed MLP network is composed of an input layer, 
an output layer and one hidden layer. The number of neurons in the input layer is equal to the number of independent variables, i.e. 5 neurons, while the number of output neurons should be equal to the numbers of dependent variable to be detected (i.e. relative crystallinity). Therefore, the network's input and output consist of a five- and one-element vector, respectively. Each layer is interconnected to the subsequent layer and the strength of these connections is determined by their associated parameters, i.e. weights. The weights and biases can be adjusted by training the network using the standard back propagation algorithm.

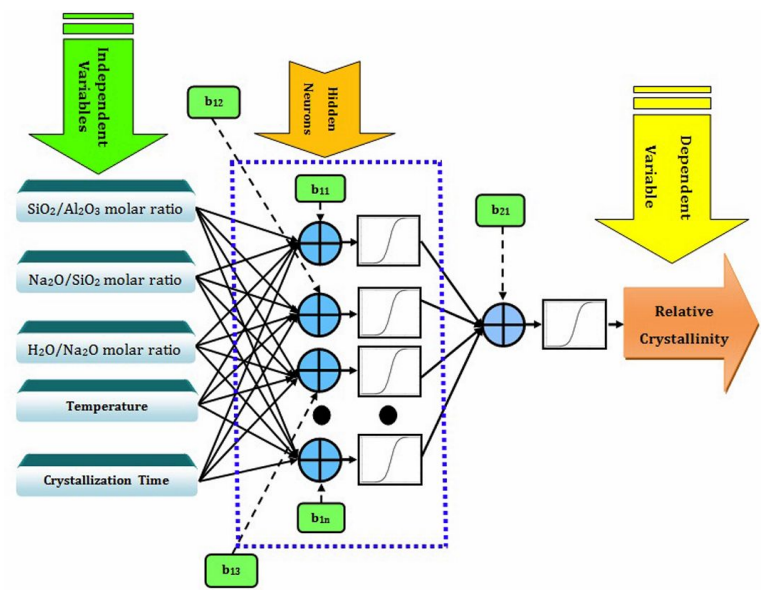

Fig. 1. Architecture of the proposed ANN model $\left(b_{m n}\right.$ is the bias of $\mathrm{n}^{\text {th }}$ neurons in the $\mathrm{m}^{\text {th }}$ layer).

Our previous findings $[29,30,33-35,40]$ justified that designing the MLP model from normalized data is easier than working on the original data. Therefore, in the present study all the variables have been mapped between [0 1] intervals. As it can be seen from Fig. 1, in the present study the log-sigmoid is utilized as the transfer function in the input as well as output layers.

\subsection{Experimental}

At the first stage of our study, 128 experimental measurements for the degree of relative crystallinity of LTA zeolites have been collected by either doing experiments or from the literature $[10,12,20-23]$. Our data-bank covers the $\mathrm{SiO}_{2}: \mathrm{Al}_{2} \mathrm{O}_{3}$ ratio 1.1:25, $\mathrm{Na}_{2} \mathrm{O}: \mathrm{SiO}_{2}$ ratio 0.03:8.1, $\mathrm{H}_{2} \mathrm{O}: \mathrm{Na}_{2} \mathrm{O}$ ratio 20:340, temperature ranges of
$60{ }^{\circ} \mathrm{C}$ to $110^{\circ} \mathrm{C}$, and crystallization time of $0.5 \mathrm{~h}$ to $20 \mathrm{~h}$. These experimental data have been employed for designing the proposed MLP network and validation its predictive accuracy. The collected experimental data, zeolite synthesis ingredients, their considered ranges and operating conditions, as well as minimum-maximum values of the available experimental observations are summarized in Table 1.

\subsection{Determining an optimum configura- tion of MLP approach}

The experimental data have been randomly divided into training and testing groups. Training subset is employed for adjusting unknown parameters of ANN approach (weights and biases). Thereafter, the predictive capability of the trained ANN model is examined and validated by testing the dataset. Various ANN types such as RBF, MLP, $\mathrm{CFB}$ and GR neural networks are checked and the best one is determined through comparison of their predictive accuracies.

Although predictive performance of larger ANN for the training dataset is better than the small ones, the former often encounters severe difficulty for prediction of the testing subset. Therefore, an optimum size of the ANN approach (number of hidden neurons) is the smallest network than can present an acceptable accuracy for estimation of both the training and testing subsets. The predictive accuracy of ANN model can be numerically determined by some statistical error indices, such as mean square error (MSE), absolute average relative deviation percent (AARD \%), and regression coefficient $\mathrm{R}^{2}$. These statistical error indices can be mathematically expressed by equation 4 through equation 6 , respectively.

$$
\begin{gathered}
M S E=\frac{1}{N} \sum_{i=1}^{N}\left(R C_{i}^{\text {exp. }}-R C_{i}^{\text {calc. }}\right)^{2} \\
A A R D \%=\frac{1}{N} \sum_{i=1}^{N}\left(\left|\frac{R C_{i}^{\text {exp }}-R C_{i}^{\text {calc. }}}{R C_{i}^{\text {exp. }}}\right|\right) \times 100
\end{gathered}
$$


Table 1. Physical and operational conditions of various datasets.

\begin{tabular}{cccccccc}
\hline $\mathrm{SiO}_{2}: \mathrm{Al}_{2} \mathrm{O}_{3}$ & $\mathrm{Na}_{2} \mathrm{O}: \mathrm{SiO}_{2}$ & $\mathrm{H}_{2} \mathrm{O}: \mathrm{Na}_{2} \mathrm{O}$ & $\mathrm{T}^{\mathrm{a}}\left[{ }^{\circ} \mathrm{C}\right]$ & $\mathrm{CT}^{\mathrm{b}}[\mathrm{h}]$ & $\mathrm{RC}^{\mathrm{c}}$ & $\mathrm{N}^{\mathrm{d}}$ & The literature \\
\hline \hline $2-10$ & $0.46-2.9$ & $39-91$ & $60-110$ & $5-15$ & $10-100$ & 13 & this work \\
$2-25$ & $0.03-3$ & $20-340$ & $90-150$ & $2-20$ & $1-100$ & 5 & {$[10]$} \\
$1.1-2.17$ & $2.25-8.1$ & $39.4-56.5$ & $73-95$ & $0.5-7.5$ & $14-95$ & 13 & {$[12]$} \\
1.64 & 8.1 & 56.5 & $90-95$ & $0.5-3.5$ & $14-67$ & 14 & {$[20]$} \\
$1.1-2.17$ & $2.25-3.03$ & $39.4-51.2$ & $73-80$ & $5.18-7.4$ & $58.5-95.2$ & 24 & {$[21]$} \\
2 & 1.3 & 50 & $60-90$ & $1-8$ & $33-91$ & 43 & {$[22]$} \\
1.94 & 2.06 & 25 & $60-70$ & $2.17-10$ & $1-93$ & 16 & {$[23]$} \\
\hline
\end{tabular}

${ }^{\mathrm{a}}$ Temperature

${ }^{\mathrm{b}}$ Crystallization time

${ }^{\mathrm{c}}$ Relative crystallinity

${ }^{\mathrm{d}}$ Number of experimental data

$$
R^{2}=\frac{\sum_{i=1}^{N}\left(R C_{i}^{\text {exp }}-\overline{\Delta R C}\right)^{2}-\sum_{i=1}^{N}\left(R C_{i}^{\text {exp. }}-\Delta R C_{i}^{\text {calc. }}\right)^{2}}{\sum_{i=1}^{N}\left(R C_{i}^{\text {exp }}-\overline{\Delta R C}\right)^{2}}
$$

where $\mathrm{N}$ represents the number of experimental data, relative experimental crystallinity $R C_{i}^{e x p}$ is the experimental value of relative crystallinity, and relative calculated crystallinity $\mathrm{RC}_{\mathrm{i}}^{\text {calc. }}$ is the value of relative crystallinity predicted by the developed MLP model. Average value of the data of relative crystallinity is shown by $\overline{\Delta \mathrm{RC}}$.

At the first step, an optimum number of hidden neurons of the MLP model is determined, and then its predictive accuracy is benchmarked using those experimental data which are not used through the training step. The same procedure is employed for evaluating an optimal topology of the other considered ANN models, i.e. RBF, GR and CFB approaches.

Table 2 presents values of the MSE, AARD \% and $\mathrm{R}^{2}$ of different MLP networks differing by the number of hidden neurons for training, testing as well as overall dataset (training + testing). It should be mentioned that the only difference among these MLP networks is the number of their hidden neurons.

The reported values of the error indices in Table 2 clearly confirm that the MLP network with eight hidden neurons (the bold row) which estimates all the experimental data with an excellent
Table 2. Performed sensitivity analysis to evaluate an optimal MLP configuration.

\begin{tabular}{|c|c|c|c|c|}
\hline \multirow{2}{*}{$\begin{array}{l}\text { Hidden } \\
\text { neurons }\end{array}$} & \multirow{2}{*}{ Dataset } & \multicolumn{3}{|c|}{ Statistical accuracy analysis* } \\
\hline & & AARD $\%$ & MSE & $\mathrm{R}^{2}$ \\
\hline \multirow{3}{*}{4} & Train & 10.38 & $7.50 \times 10^{-6}$ & 0.919733 \\
\hline & Test & 32.29 & $2.02 \times 10^{-5}$ & 0.883313 \\
\hline & Overall & 11.40 & $8.09 \times 10^{-6}$ & 0.915907 \\
\hline \multirow{3}{*}{6} & Train & 9.80 & $4.36 \times 10^{-6}$ & 0.956004 \\
\hline & Test & 12.1 & $1.73 \times 10^{-5}$ & 0.804316 \\
\hline & Overall & 11.99 & $4.97 \times 10^{-6}$ & 0.949246 \\
\hline \multirow{3}{*}{7} & Train & 8.81 & $3.01 \times 10^{-6}$ & 0.969932 \\
\hline & Test & 12.04 & $1.89 \times 10^{-5}$ & 0.593392 \\
\hline & Overall & 10.87 & $3.76 \times 10^{-6}$ & 0.962130 \\
\hline \multirow{3}{*}{8} & Train & 8.23 & $3.89 \times 10^{-6}$ & 0.960577 \\
\hline & Test & 8.71 & $5.87 \times 10^{-6}$ & 0.949055 \\
\hline & Overall & 8.69 & $3.99 \times 10^{-6}$ & 0.959624 \\
\hline \multirow{3}{*}{9} & Train & 6.83 & $2.77 \times 10^{-6}$ & 0.972476 \\
\hline & Test & 11.4 & $3.54 \times 10^{-6}$ & 0.968016 \\
\hline & Overall & 11.19 & $2.81 \times 10^{-6}$ & 0.971683 \\
\hline \multirow{3}{*}{10} & Train & 5.22 & $2.44 \times 10^{-6}$ & 0.976019 \\
\hline & Test & 12.17 & $1.16 \times 10^{-5}$ & 0.746325 \\
\hline & Overall & 5.55 & $2.87 \times 10^{-6}$ & 0.970968 \\
\hline \multirow{3}{*}{12} & Train & 4.68 & $2.25 \times 10^{-6}$ & 0.973564 \\
\hline & Test & 14.59 & 0.000138 & 0.452852 \\
\hline & Overall & 18.08 & $8.61 \times 10^{-6}$ & 0.911721 \\
\hline
\end{tabular}

${ }^{*}$ Only the best obtained values over twenty different trainings per each topology are tabulated.

accuracy (MSE and AARD \% of $3.99 \times 10^{-6}$ and $8.69 \%$, respectively) is an optimum topology. The optimal MLP model shows the $\mathrm{R}^{2}$ value 
of 0.959624 for prediction of experimental data of crystallinity of the LTA zeolites. Values of the statistical indices approve the excellent agreement between experimental data of relative crystallinity of various LTA zeolites and their associated predicted values by the developed MLP model.

In order to simplify the comparison among prediction capabilities of various MLP networks (with different numbers of hidden neurons), their AARD \% over the training and testing categories is illustrated in Fig. 2.

It can be simply understood from Fig. 2 that, by increasing the number of hidden neurons of the MLP network, the value of AARD \% continuously decreases for training dataset. But the AARD \% of estimation for the testing dataset decreases until eight hidden neurons, and thereafter no improvement in AARD \% is obtained for the testing group. Therefore, employing more than eight hidden neurons over-fits the MLP network over training dataset, and fails in prediction of the testing dataset. Consequently, the two-layer network with eight hidden neurons is chosen as an optimal topology for the prediction of degree of relative crystallinity of the LTA zeolites.

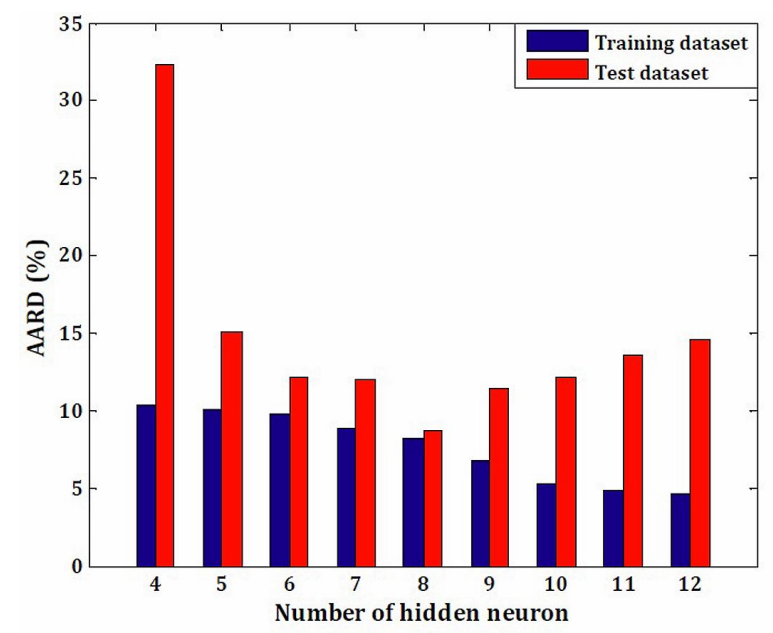

Fig. 2. Associated AARD \% of various MLP topologies over test and training subsets.

Variation of the mean square error for the best MLP network versus epoch during its training process is depicted in Fig. 3. The y-axis of this figure

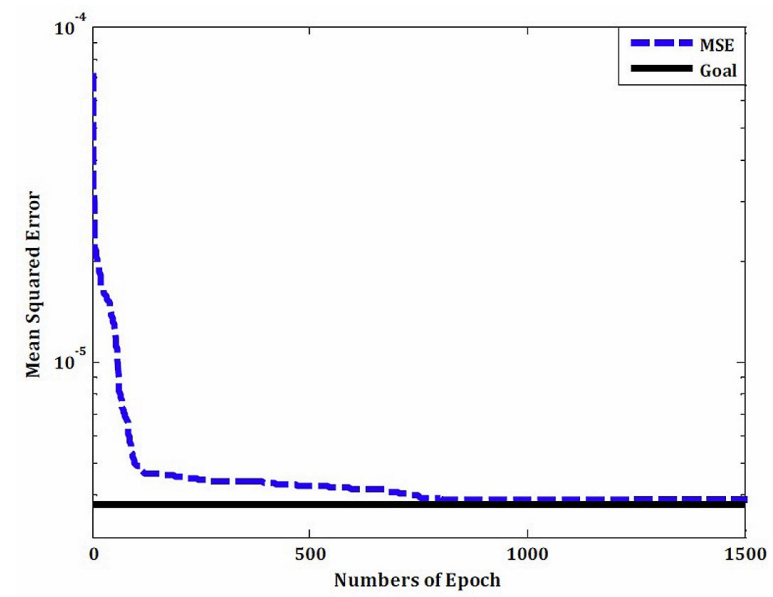

Fig. 3. MSE variation versus epoch for the optimal MLP predicting relative crystallinity; solid line represents the goal while dashed line is training MSE.

represents the values of observed MSE between the optimal real target and MLP prediction, whereas the $\mathrm{x}$-axis illustrates an epoch. The epoch refers to the number of times that the training dataset is fed to the MLP model for adjusting its parameters.

The MLP outputs of the first epoch calculated through performing some mathematical processes on the raw independent variables are based on the procedure described in section 2.2.1. Since the initial values of the weight and bias are randomly generated, therefore some deviations (large extent of MSE) are often observed between MLP outputs and the real targets at the first epoch. The observed error can be employed for updating the weights and biases during the training process. These updated weights and biases are used in the second epoch. Training process continues until the MSE converges to its predetermined values.

By increasing the number of epochs, the weights and biases of the MLP model converge to their optimal values and hence, the observed MSE between MLP prediction and real target decreases. It can be seen from Fig. 3 that the observed MSE value between observed and calculated values by the MLP network converges to the acceptable values of $3.9 \times 10^{-6}$ after 1500 epochs and its learning process seems to be successful. 


\subsection{Comparison of the predictive accu- racy of various ANN models}

In this section, the predictive accuracy of the MLP network has been compared with the other ANN types, i.e. RBF, GR and CFB. The results of comparison analysis between the performances of the developed MLP network for prediction of relative crystallinity of the LTA zeolites with the other ANN approaches are presented in Table 3. In order to base the comparison on a fair basis, eight neurons have been put in the hidden layer of all the considered ANN types (RBF, GR and CFB neural network).

These comparison studies have been accomplished based on the AARD \%, MSE and $\mathrm{R}^{2}$. It can be simply understood that a network with the smallest AARD \% and MSE, and $\mathrm{R}^{2}$ closer to unity should be selected as the best model for the considered task. The MLP, GR, RBF and CFB neural networks show the AARD \% of $8.69 \%, 29.73 \%$, $35.72 \%$ and $10.89 \%$ for estimation all the experimental relative crystallinity, respectively. It is obvious that the RBF model presents the minimum error $(\mathrm{AARD}=1.03 \%)$ for the training dataset while the highest error for training dataset $(\mathrm{AARD}=12.55 \%)$ is introduced by the GR network. Comparison of the performance efficiencies of the four different ANN types clears that the MLP network (the bold row) is the best approach for estimating the values of relative crystallinity of the LTA zeolites.

While the two-layer MLP network with eight hidden neurons introduces the best capability for estimation and simulation of relative crystallinity of the LTA zeolites, it can be considered as the optimal neural network method.

\subsection{Prediction accuracy of the optimal MLP model over experimental data}

Fig. 4 shows the comparison between those values of relative crystallinity of the LTA zeolites which are predicted by the optimal neural network and their associated experimental training and testing datasets. The perfect fit (predictions equal with the real
Table 3. Comparison the predictive accuracy of various ANNs approaches.

\begin{tabular}{llccc}
\hline \multirow{2}{*}{$\begin{array}{c}\text { ANN } \\
\text { type }\end{array}$} & & \multicolumn{3}{c}{ Statistical sensitivity analysis } \\
\cline { 2 - 5 } MLP & AARD \% & MSE & $\mathrm{R}^{2}$ \\
\hline \hline \multirow{3}{*}{ MLP } & Training Dataset & 8.23 & $3.89 \times 10^{-6}$ & 0.960577 \\
\cline { 2 - 5 } & Testing Dataset & 8.71 & $5.87 \times 10^{-6}$ & 0.949055 \\
\cline { 2 - 5 } & Overall Dataset & 8.69 & $3.99 \times 10^{-6}$ & 0.959624 \\
\hline \multirow{3}{*}{ GBF } & Training Dataset & 12.55 & $3.21 \times 10^{-5}$ & 0.936425 \\
\cline { 2 - 5 } & Testing Dataset & 61.70 & 4.50343 & 0.423053 \\
\cline { 2 - 5 } & Overall Dataset & 29.73 & 2.24124 & 0.823536 \\
\hline \multirow{3}{*}{ CFB } & Training Dataset & 1.03 & $1.09 \times 10^{-7}$ & 0.996425 \\
\cline { 2 - 5 } & Testing Dataset & 82.62 & 4.83243 & 0.403123 \\
\hline & Overall Dataset & 35.72 & 2.47360 & 0.783124 \\
\cline { 2 - 5 } & Training Dataset & 9.31 & $8.09 \times 10^{-6}$ & 0.953512 \\
\cline { 2 - 5 } & Testing Dataset & 13.20 & $3.90 \times 10^{-5}$ & 0.943578 \\
\hline
\end{tabular}

data) is shown by the dashed $45^{\circ}$ line. The small deviation from the $45^{\circ}$ line in Fig. 4, shows that the predicted training and testing subsets have been correctly mapped on their associated experimental values.

The optimal MLP approach presents $\mathrm{R}^{2}=0.9605$ between the predictions and experimental relative crystallinity data of the training dataset. The proposed model has predicted the experimental relative crystallinity data of the training subset with AARD \% and MSE of $8.23 \%$, $3.89 \times 10^{-6}$, respectively. It should be noted that the optimal MLP network introduces MSE = $5.87 \times 10^{-6}$, AARD $\%=8.71$ and $\mathrm{R}^{2}=0.9490$ for estimation of the testing subset.

Fig. 5 indicates graphical presentation of the AARD \% values which have been introduced by the optimal MLP approach for estimation our data as well as reported experimental datasets by other groups of researchers [10, 12, 20, 23]. It can be clearly seen from this figure that the proposed MLP model encounters some difficulties (relatively large AARD \%) in prediction the behavior of the reported data by Murat et al. [22] and Park et al. [23] and presents the AARD \% of $10.2 \%$ and $12.2 \%$ for these two datasets, respectively. Excluding these two datasets, the developed MLP approach shows remarkable predictive accuracy in modeling other available experimental data and estimates 


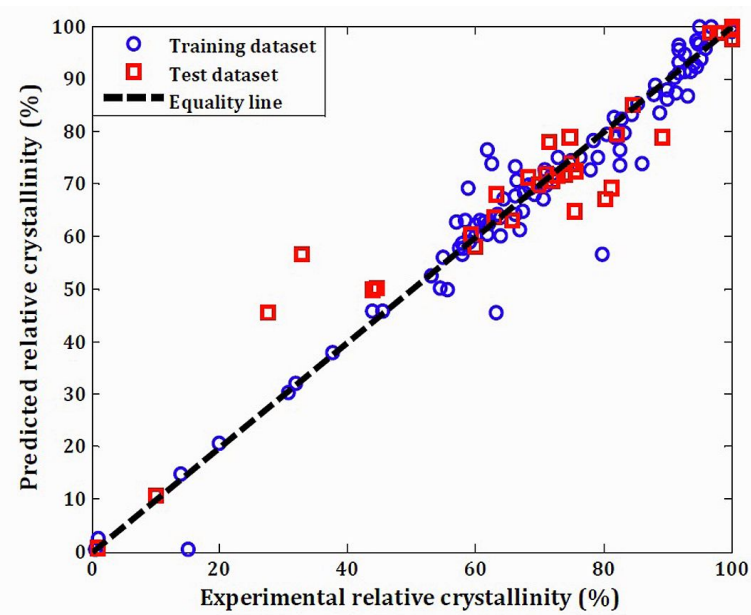

Fig. 4. Schematic presentation of performance of the optimal MLP network for estimation the degree of relative crystallinity of LTA zeolites over training + test datasets.

all of them with overall AARD \% lower than $3 \%$.

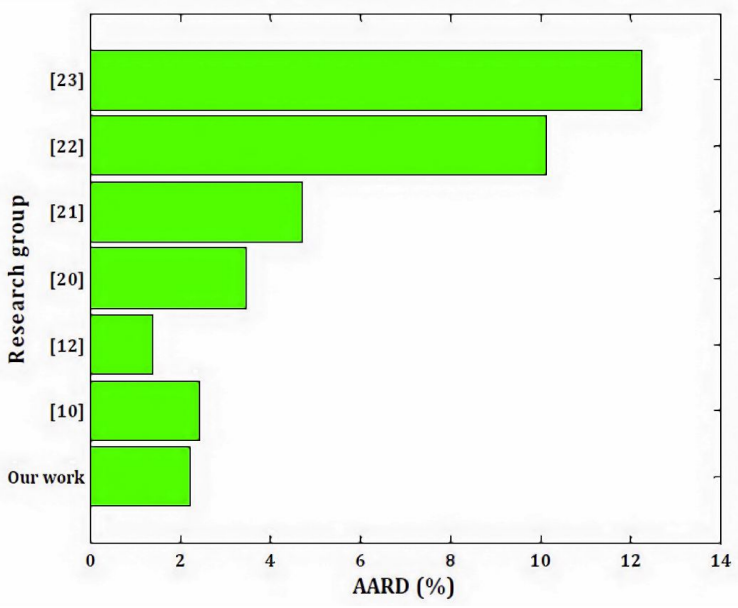

Fig. 5. The performance of the MLP network with optimal topology for prediction the reported relative crystallinity by various groups of researchers.

\subsubsection{Investigation of the effect of crystalliza- tion time on the relative crystallinity}

Fig. 6 illustrates the variation of degree of relative crystallinity for the LTA zeolite as a function of crystallization time. The experimental values of crystallinity for the LTA zeolite in this figure are either our experimental data or other researcher results $[10,12,20,23]$. It can be concluded that increasing the crystallization time can increase the extent of relative crystallinity for the LTA zeolites. In order to ease the comparison between experimental data of crystallinity for the LTA zeolite and calculated values by the best MLP network, the results of MLP network are also presented in Fig. 6. It can be clearly concluded that the proposed MLP model can correctly track the increasing pattern of crystallinity for the LTA zeolite with time as well as estimate all the individual experimental data.

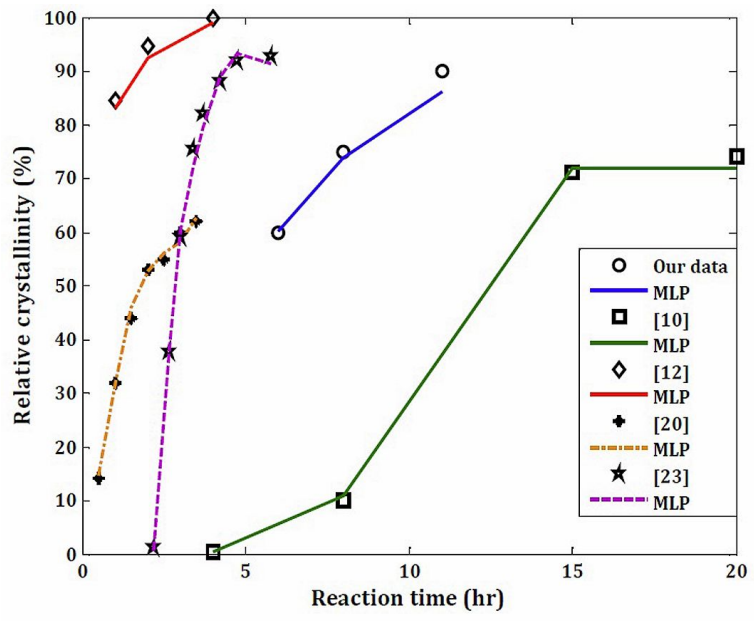

Fig. 6. Experimental and theoretical investigation of the influence of reaction time on the extent of relative crystallinity of the LTA zeolites.

\subsubsection{Variation of the extent of relative crys- tallinity by temperature}

Fig. 7 depicts variation of relative crystallinity for the LTA zeolites as a function of operating temperature $[12,23]$. It can be simply understood from Fig. 7, that increasing the temperature can result in increasing the extent of relative crystallinity for the LTA zeolites. The relative crystallinity values predicted by the optimal MLP methodology, which are also presented in Fig. 7, confirm the excellent agreement between MLP predictions and experimental data points. It can be clearly observed that the designed MLP approach can correctly follow the rising trend as well as estimate all the individual experimental data. 


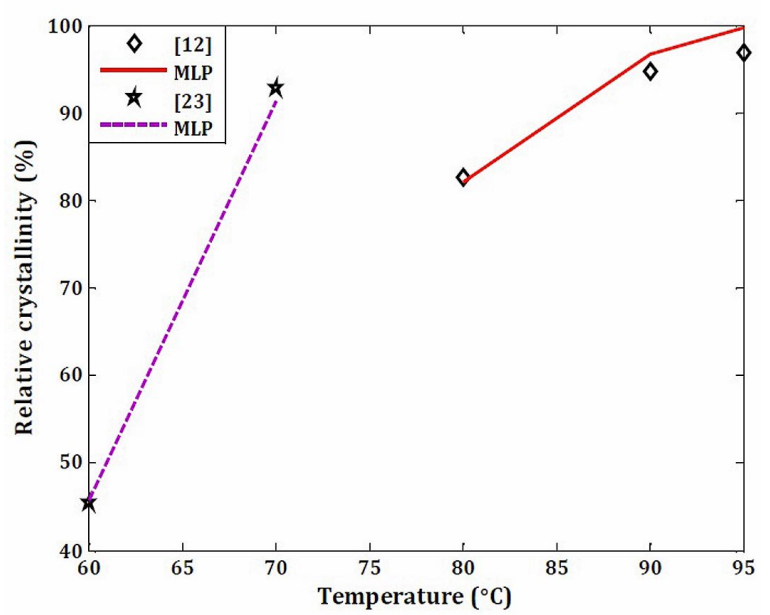

Fig. 7. Profile of degree of relative crystallinity of the LTA zeolites as a function of temperature and values predicted by the optimal MLP network.

\subsubsection{Effect of $\mathrm{H}_{2} \mathrm{O}: \mathrm{Na}_{2} \mathrm{O}$ molar ratio on the degree of relative crystallinity}

In Fig. 8, experimental values of relative crystallinity of the LTA zeolite which have been obtained by our own experiments or reported by other researchers [12, 21], as well as their associated predictions which have been simulated by the optimal MLP network, are plotted as a function of the molar ratio of $\mathrm{H}_{2} \mathrm{O}: \mathrm{Na}_{2} \mathrm{O}$.

Fig. 8 clearly illustrates that the proposed MLP approach can forecast both increasing and decreasing pattern for variation of experimental values of relative crystallinity for the LTA zeolite at different molar ratios of $\mathrm{H}_{2} \mathrm{O}: \mathrm{Na}_{2} \mathrm{O}[12,21]$.

\subsubsection{Investigation of the effect of $\mathrm{SiO}_{2}: \mathrm{Al}_{2} \mathrm{O}_{3}$ and $\mathrm{Na}_{2} \mathrm{O}: \mathrm{SiO}_{2}$ molar ratios}

The ratio of $\mathrm{SiO}_{2}: \mathrm{Al}_{2} \mathrm{O}_{3}$ has a slight influence on the degree of crystallinity of the LTA zeolite. In general, it was one of the most decisive parameters but because of the present focus on LTA preparation it was already biased towards its formation. However, some researchers have observed that the degree of crystallinity increases for lower $\mathrm{SiO}_{2}: \mathrm{Al}_{2} \mathrm{O}_{3}$ molar ratios reaching maximum value for $\mathrm{SiO}_{2}: \mathrm{Al}_{2} \mathrm{O}_{3} \approx 1$ [49]. Moreover, Burriesci et al. [49] have reported that by increasing the molar ratio of $\mathrm{Na}_{2} \mathrm{O}: \mathrm{SiO}_{2}$, different types

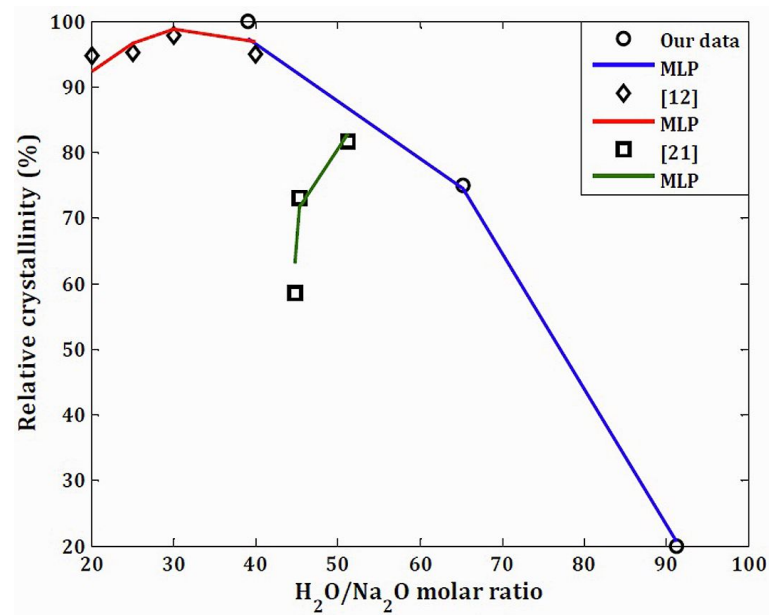

Fig. 8. Experimental values of relative crytallinty of the LTA zeolite as well as their associated predictions by the MLP network versus $\mathrm{H}_{2} \mathrm{O}: \mathrm{Na}_{2} \mathrm{O}$ molar ratio.

of zeolites including LTA, sodalite, phillipsite and faujasite may be produced.

\section{Conclusions}

In the present study, an automatic intelligent methodology based on artificial neural network is designed for mathematical simulation of the extent of relative crystallinity for LTA zeolites. Some easily measured variables, i.e. molar ratios of the $\mathrm{SiO}_{2}: \mathrm{Al}_{2} \mathrm{O}_{3}, \mathrm{Na}_{2} \mathrm{O}: \mathrm{SiO}_{2}$ and $\mathrm{H}_{2} \mathrm{O}: \mathrm{Na}_{2} \mathrm{O}$ in the reaction mixture, temperature and crystallization time are employed as independent variables for the considered task. Iterative constructive method combined with statistical error analyses reveal that the two-layer perceptron network (strictly feed-forward structure) with eight hidden neurons is the best approach for simulation the degree of crystallinity for the LTA zeolite. Performance of the best developed MLP approach has been benchmarked by both our experimental data, and some reported experimental data from other researchers. The results of the developed MLP network confirm that it is capable to predict the degree of relative crystallinity for the LTA zeolites with an excellent accuracy $\left(\mathrm{MSE}=3.99 \times 10^{-6}\right.$, AARD \% $=8.69$ and $\left.\mathrm{R}^{2}=0.9596\right)$. Excellent performance of the designed MLP network in prediction 
of the experimental data confirms that this approach can be effectively employed for accurate estimation of relative crystallinity for LTA zeolites.

\section{References}

[1] GuO Y.H., Pu M., LiU L.Y., Li H.F., CHEN B.H., Comp. Mater. Sci., 42 (2008), 179.

[2] Benco L., Demuth T., HutschKa F., Comp. Mater. Sci., 27 (2003), 87.

[3] Chong S.X., Wahab H.A., Abdallah H.H., Comp. Mater. Sci., 55 (2012), 217.

[4] Hou J., Yuan J., Shang R., Powder. Technol., 226 (2012), 222.

[5] Naskar M.K., Das A., Kundu D., ChatterJEE M., B. Mater. Sci., 34 (2011), 651.

[6] Shang J., Li G., Webley P.A., LiU J.Z., Comp. Mater. Sci., 122 (2016), 307.

[7] Mahadwad O.K., PARIKH P.A., JASRa R.V., PATIL C., B. Mater. Sci., 34 (2011), 551.

[8] Charkhi A., Kazemeini M., Ahmadi S.J., KAZEMian H., Powder. Technol., 231 (2012), 1.

[9] Kazemian H., Modarress H., KaZemi M., FARHADI F., Powder. Technol., 196 (2009), 22.

[10] Kim D.S., Chang J.S., Hwang J.S., Park S.E., KIM J.M., Micropor. Mesopor. Mat., 68 (2004), 77.

[11] Elnekave M., Tatlier M., Chem. Eng. Commun., 195 (2008), 661.

[12] LiU X.D., Wang Y.P., Cui X.M., He Y., MaO J., Powder. Technol., 243 (2013), 184.

[13] Bosnar S., Bronic J., Brlek D., Subotic B., Micropor. Mesopor. Mat., 142 (2011), 389.

[14] Ciric J., J. Colloid. Interf. Sci., 28 (1968), 315.

[15] Krznaric I., Antonic T., Subotic B., BabiCIVANCIC V., Thermochim. Acta, 317 (1998), 73.

[16] Bosnar S., Antonic-Jelic T., Bronic J., KrZNARIC I., Subotic B., J. Cryst. Growth, 267 (2004), 270.

[17] CIRIC J., Science, 155 (1967), 689.

[18] Tanaka H., Fujil A., Fujimoto S., Tanaka Y., Powder. Technol., 19 (2008), 83.

[19] Ansari M., Aroujalian A., Raisi A., Dabir B., Fathizadeh M., Adv. Powder. Technol., 25 (2014), 722.

[20] Chauhan Y.P., Talib M., Sci. Rev. Chem. Ccommun., 2 (2012), 12.

[21] Garcia-Seto A.R., Rodriguez-Nino G., TruJILLO C.A., Ing. Invest., 33 (2013), 22.

[22] Murat M., Amokrane A., Bastide J.P., MontaNARO L., Clay Miner., 27 (1992), 119.

[23] PARK J., Kim B.C., PARK S.S., PARK H.C., J. Mater. Sci. Lett., 20 (2001), 531.
[24] Herrmann R., Schwieger W., Scharf O., StenZal C., Toufar H., Zchmachtl M., Ziberi B., GRILL W., Micropor. Mesopor. Mat., 80 (2005), 1.

[25] Thompson R.W., Franklin K.C., Linde Type A, in: H. RobSON (Ed.), Verified Synthesis of Zeolititic Materials, Elsevier, Amesterdam, 2001.

[26] Tosheva L., Valtchev V.P., Chem. Mater, 17 (2005), 2494.

[27] Chen J., Yea Y., Chem. Eng. Commun., 189 (2002), 865.

[28] Ko Y.D., Shang H., Powder. Technol., 205(2011), 250.

[29] LASHKarbolooki M., VAFERI B., RAHIMPOUR M.R., Fluid Phase Equilibr., 308 (2011), 35.

[30] Lashkarbolooki M., Vaferi B., Shariati A., Zeinolabedini Hezave A., Fluid Phase Equilibr., 343 (2013), 24.

[31] Artrith N., Urban A., Comp. Mater. Sci., 114 (2016), 135.

[32] Castin N., Fernandez J.R., Pasianot R.C., Comp. Mater. Sci., 84 (2014), 217.

[33] Vaferi B., Eslamloueyan R., Ayatollahi S., $J$. Petrol. Sci. Eng., 77 (2011), 254.

[34] Vaferi B., Karimi M., Azizi M., Esmaeili H., J. Supercrit. Fluid., 77 (2013), 44.

[35] VAFERI B., RAHNAMA Y., DARVishi P., TOORANI A., LASHKARBOLOOKI M., J. Supercrit. Fluid., 84 (2013), 80.

[36] JaCK L.B., NANDi A.K., Mech. Syst. Signal Pr., 16 (2002), 373.

[37] Sun Y., Zeng W.D., Han Y.F., Ma X., ZhaO Y.Q., Comp. Mater. Sci., 50 (2011), 1064.

[38] Mohammad A.T., Mat S.B., Sulaiman M.Y., Sopian K., Al-ABIDI A.A., Energ. Convers. Manage., 67 (2013), 240.

[39] Elman J.L., Cognitive Sci., 14 (1990), 179.

[40] Vaferi B., Samimi F., Pakgohar E., Mowla D., Powder. Technol., 267 (2014), 1.

[41] Elish M.O., Expert Syst. Appl., 36 (2009), 10774.

[42] SPECHT D.F., IEEE T. Neur. Net., 2 (1991), 568.

[43] Hopfield J.J., Proc. Nat. Acad. Sci., 79 (1982), 2554.

[44] Cybenko G.V., Math. Control Signal, 2 (1989), 303.

[45] Funahashi K.I., Neural Networks, 2 (1989), 183.

[46] Hornik K., Stinchcombe M., White H., Neural Networks, 2 (1989), 359.

[47] DU K.L., SWAmy M.N.S., Neural Networks in a Soft computing Framework, Springer, London, 2006.

[48] REED R., IEEE T. Neur. Net., 4 (1993), 740.

[49] Burriesci N., Crisafulli M.L., Mater. Lett., 2 (1984), 401.

Received 2016-10-08 Accepted 2017-02-24 